Bull. Korean Math. Soc. 49 (2012), No. 6, pp. 1147-1161

http://dx.doi.org/10.4134/BKMS.2012.49.6.1147

\title{
COINCIDENCE THEOREMS FOR NONCOMPACT RE-MAPS IN ABSTRACT CONVEX SPACES WITH APPLICATIONS
}

\author{
Ming-Ge YANG AND NAN-Jing HuANG
}

\begin{abstract}
In this paper, a coincidence theorem for a compact $\mathfrak{R} \mathfrak{C}$-map is proved in an abstract convex space. Several more general coincidence theorems for noncompact $\mathfrak{R C}$-maps are derived in abstract convex spaces. Some examples are given to illustrate our coincidence theorems. As applications, an alternative theorem concerning the existence of maximal elements, an alternative theorem concerning equilibrium problems and a minimax inequality for three functions are proved in abstract convex spaces.
\end{abstract}

\section{Introduction}

Many problems in nonlinear analysis can be solved by showing the intersection of certain family of subsets of an underlying set is nonempty. The first remarkable result on the nonempty intersection was the celebrated KnasterKuratowski-Mazurkiewicz theorem (simply, the KKM principle) in 1929 [11], which concerns with certain types of maps called KKM maps later.

At the beginning, the KKM theory was mainly devoted to the study of convex subsets of topological vector spaces. Later it has been extended to convex spaces by Lassonde [12], to $C$-spaces (or $H$-spaces) by Horvath $[8,9]$, and to generalized convex $(G$-convex) spaces by Park and Kim [24] and Park $[21,22,23]$. Recently, Park [16] introduced a new concept of abstract convex spaces which include convex subsets of topological vector spaces, convex spaces, $C$-spaces and $G$-convex spaces as special cases. Park [16] also introduced certain broad classes $\mathfrak{R O}$ and $\mathfrak{R C}$ of maps (having the KKM property), which includes the well-known class $K K M(X, Y)$ introduced by Chang and Yen [5] as a special case. With these new concepts, some coincidence theorems and fixed point

Received June 1, 2009.

2010 Mathematics Subject Classification. 54H25, 49J53, 49J35.

Key words and phrases. coincidence theorem, alternative theorem, minimax inequality, abstract convex space, $\mathfrak{R C}$-map.

This work was supported by the Key Program of NSFC (Grant No. 70831005), the National Natural Science Foundation of China (No. 11171237), the Science and Technology Program Project of Henan Province of China (No. 122300410256) and the Natural Science Foundation of Henan Education Department of China (No. 2011B110025). 
theorems were proved in abstract convex spaces by Park [16]. Very recently, Park [17, 18, 19, 20] further studied KKM theory in abstract convex spaces with applications to fixed points, maximal elements, equilibria problems and other problems. It is noted that, in the KKM theory, there have appeared a number of coincidence theorems with many significant applications.

On the other hand, since Fan [7] and Liu [15] extended the von NeumannSion principle obtaining two-function minimax inequalities, many such results involving two or more functions have been obtained in the literature (see, for example, $[1,3,18]$ and the references therein).

Motivated and inspired by the works mentioned above, in this paper, we first obtain a coincidence theorem for a compact $\mathfrak{R C}$-map in abstract convex space. Then we derive several more general coincidence theorems for noncompact $\mathfrak{R C}$ maps in abstract convex spaces. We also give some examples to illustrate our coincidence theorems. As applications, we prove an alternative theorem concerning the existence of maximal elements, an alternative theorem concerning equilibrium problems, and a three-function minimax inequality of the following type:

$$
\inf _{x \in X} h(x, x) \leq \sup _{x \in X} \inf _{z \in Z} f(x, z)+\sup _{z \in Z} \inf _{x \in X} g(x, z)
$$

in abstract convex spaces. The results presented in this paper improve and generalize some corresponding results due to Balaj [1], Balaj and Lin [3] and Lin, Ansari and Wu [14].

\section{Preliminaries}

A multimap (or simply a map) $T: X \multimap Y$ is a function from a set $X$ into the power set $2^{Y}$ of $Y$, that is a function with the values $T(x) \subset Y$ for $x \in X$. Given a map $T: X \multimap Y$, the map $T^{-}: Y \multimap X$ defined by $T^{-}(y)=\{x \in$ $X: y \in T(x)\}$ for $y \in Y$, is called the (lower) inverse of $T$. For any $A \subset X$, $T(A):=\bigcup_{x \in A} T(x)$. For any $B \subset Y, T^{-}(B):=\{x \in X: T(x) \cap B \neq \emptyset\}$. For a set $X,\langle X\rangle$ will denote the family of all nonempty finite subsets of $X$.

If $A$ is a subset of a topological space, we denote by $\operatorname{int} A$ and $\bar{A}$ the interior and closure of $A$, respectively.

For topological spaces $X$ and $Y$, a map $T: X \multimap Y$ is said to be compact if $T(X)$ is contained in a compact subset of $Y$. Let $\bar{T}: X \multimap Y$ be a map defined by $\bar{T}(x)=\overline{T(x)}$ for $x \in X$.

Definition 2.1 ([25]). Suppose that $X$ is a nonempty set and $Y$ is a topological space. A map $T: X \multimap Y$ is said to be transfer open valued if for any $(x, y) \in X \times Y$ with $y \in T(x)$ there exists an $x^{\prime} \in X$ such that $y \in \operatorname{int} T\left(x^{\prime}\right)$.

Note that a map with open values is transfer open valued, but generally the converse is not true.

Lemma 2.1 ([13]). Let $X$ be a topological space, $Z$ be a nonempty set, and $P: X \multimap Z$ be a map. Then the following assertions are equivalent: 
(i) $P^{-}$is transfer open valued and $P$ has nonempty values;

(ii) $X=\bigcup_{z \in Z}$ int $P^{-}(z)$.

Definition 2.2 ([16]). An abstract convex space $(E, D ; \Gamma)$ consists of a nonempty set $E$, a nonempty set $D$, and a map $\Gamma:\langle D\rangle \multimap E$ with nonempty values. We denote $\Gamma_{A}:=\Gamma(A)$ for $A \in\langle D\rangle$.

An abstract convex space $(E, D ; \Gamma)$ with any topology on $E$ is called an abstract convex topological space.

In case $E=D$, let $(E ; \Gamma):=(E, E ; \Gamma)$. It is obvious that any vector space $E$ is an abstract convex space with $\Gamma=$ co, where co is the convex hull in vector spaces. In specially, $(\mathbb{R} ; \mathrm{co})$ is an abstract convex space. For more examples of abstract convex spaces, we refer to $[16,17,18,19,20]$.

Let $(E, D ; \Gamma)$ be an abstract convex space. For any $D^{\prime} \subset D$, the $\Gamma$-convex hull of $D^{\prime}$ is denoted and defined by

$$
\operatorname{co}_{\Gamma} D^{\prime}:=\bigcup\left\{\Gamma_{A} \mid A \in\left\langle D^{\prime}\right\rangle\right\} \subset E
$$

(co is reserved for the convex hull in vector spaces). A subset $X$ of $E$ is called a $\Gamma$-convex subset of $(E, D ; \Gamma)$ relative to $D^{\prime}$ if for any $N \in\left\langle D^{\prime}\right\rangle$, we have $\Gamma_{N} \subset X$; that is, $\operatorname{co}_{\Gamma} D^{\prime} \subset X$. This means that $\left(X, D^{\prime} ;\left.\Gamma\right|_{\left\langle D^{\prime}\right\rangle}\right)$ itself is an abstract convex space called a subspace of $(E, D ; \Gamma)$. When $D \subset E$, the space is denoted by $(E \supset D ; \Gamma)$. In such case, a subset $X$ of $E$ is said to be $\Gamma$-convex if $\operatorname{co}_{\Gamma}(X \cap D) \subset X$; in other words, $X$ is $\Gamma$-convex relative to $D^{\prime}=X \cap D$. When $(E ; \Gamma)=(\mathbb{R} ; c o), \Gamma$-convex subsets reduce to ordinary convex subsets.

Let $(E, D ; \Gamma)$ be an abstract convex space and $Z$ a set. For a map $F: E \multimap Z$ with nonempty values, if a map $G: D \multimap Z$ satisfies

$$
F\left(\Gamma_{A}\right) \subset G(A), \forall A \in\langle D\rangle,
$$

then $G$ is called a KKM map with respect to $F$. A KKM map $G: D \multimap E$ is a KKM map with respect to the identity map $1_{E}$. A map $F: E \multimap Z$ is said to have the KKM property and called a $\Re$-map if, for any KKM map $G: D \multimap Z$ with respect to $F$, the family $\{G(y)\}_{y \in D}$ has the finite intersection property. We denote

$$
\mathfrak{R}(E, Z):=\{F: E \multimap Z \mid F \text { is a } \mathfrak{R} \text {-map }\} \text {. }
$$

Similarly, when $Z$ is a topological space, a $\mathfrak{R C}$-map is defined for closedvalued maps $G$, and a $\mathfrak{R O}$-map is defined for open-valued maps $G$. Note that if $Z$ is discrete, then three classes $\mathfrak{R}, \mathfrak{R C}$ and $\mathfrak{R O}$ are identical. Some authors use the notation $K K M(E, Z)$ instead of $\mathfrak{R C}(E, Z)$.

For every abstract convex space $(E, D ; \Gamma)$ and every nonempty set $Z$, there exists a map $F \in \mathfrak{R}(E, Z)$. In fact, for each $x \in E$, choose $F(x):=Z$ or $F(x):=\left\{z_{0}\right\}$ for some $z_{0} \in Z$. If the identity map $1_{E} \in \mathfrak{R}(E, E)$, then $f \in \mathfrak{R}(E, Z)$ for any function $f: E \rightarrow Z$. If $E$ and $Z$ are topological spaces, this holds for $\mathfrak{R C}$ or $\mathfrak{R O}$ for any continuous $f$. 
It is known that for a $G$-convex space $(E, D ; \Gamma)$, we have the identity map $1_{E} \in \mathfrak{R C}(E, E) \cap \mathfrak{R O}(E, E)$. Moreover, if $F: E \rightarrow Z$ is a continuous singlevalued map or if $F: E \multimap Z$ has a continuous selection, then it is easy to check that $F \in \mathfrak{R C}(E, Z) \cap \mathfrak{R O}(E, Z)$. For more details about classes $\mathfrak{R}$, $\mathfrak{R} \mathfrak{C}$ and $\mathfrak{R O}$, we refer to $[16,17,18,19,20]$.

From now all topological spaces will be assumed to be Hausdorff.

Lemma 2.2. Let $X$ be a topological space and $(Y ; \Gamma)$ be an abstract convex space. If $T \in \mathfrak{R C}(Y, X)$ is compact and $S: Y \multimap X$ is a KKM map with respect to $T$, then $\overline{T(Y)} \cap \bigcap_{y \in Y} \overline{S(y)} \neq \emptyset$.

Proof. Since $S$ is a KKM map with respect to $T$, we have $T\left(\Gamma_{N}\right) \subset S(N)$ for each $N \in\langle Y\rangle$. It is obvious that $T\left(\Gamma_{N}\right) \subset \overline{T(Y)}$ and $S(N) \subset \bar{S}(N)$. Hence, $T\left(\Gamma_{N}\right) \subset \overline{T(Y)} \cap \bar{S}(N)$. It follows that the map $F: Y \multimap X$ defined by

$$
F(y)=\overline{T(Y)} \cap \bar{S}(y), \forall y \in Y
$$

is a KKM map with respect to $T$. Note that $F$ has closed values. By the definition of $T \in \mathfrak{R C}(Y, X)$, we have the family $\{F(y)\}_{y \in Y}$ has the finite intersection property. Moreover, $\overline{T(Y)}$ is compact. We have the family $\{F(y)\}_{y \in Y}$ has the nonempty intersection property, that is, $\overline{T(Y)} \cap \bigcap_{y \in Y} \overline{S(y)} \neq \emptyset$. This completes the proof.

Remark 2.1. (a) If $Y$ is a convex subset of a topological vector space and $\Gamma=$ co, then Lemma 2.2 reduces to Lemma 3 in [1]; (b) By using Lemma 2.2 when $Y$ is a convex space and $\Gamma=$ co, we can easily derive Lemma 2.2 in [14].

\section{Coincidence theorems}

Theorem 3.1. Let $X$ be a topological space and $(Y ; \Gamma)$ be an abstract convex space. Let $S: X \multimap Y, Q: X \multimap Y$ and $T: Y \multimap X$ be three maps satisfying the following conditions:

(i) $X=\bigcup_{y \in Y}$ int $S^{-}(y)$;

(ii) for each $x \in X, M \in\langle S(x)\rangle$ implies $\Gamma_{M} \subset Q(x)$;

(iii) $T \in \mathfrak{R C}(Y, X)$ is compact.

Then there exists $\left(x_{0}, y_{0}\right) \in X \times Y$ such that $x_{0} \in T\left(y_{0}\right)$ and $y_{0} \in Q\left(x_{0}\right)$.

Proof. Suppose the conclusion would be false. Then for each $y \in Y$ and $x \in$ $T(y)$, we have $y \notin Q(x)$. Define a map $S^{*}: Y \multimap X$ by

$$
S^{*}(y)=X \backslash S^{-}(y), \forall y \in Y .
$$

We show that $S^{*}$ is a KKM map with respect to $T$. Suppose to the contrary that there exist a finite subset $N$ of $Y$ and a point $x \in T\left(\Gamma_{N}\right) \backslash S^{*}(N)$. By $x \in T\left(\Gamma_{N}\right)$, there exists $y \in \Gamma_{N}$ such that $x \in T(y)$, and it follows that $y \notin Q(x)$. By $x \notin S^{*}(N)$, we have $x \notin S^{*}(y)$ for each $y \in N$. It follows that $y \in S(x)$ for each $y \in N$ and so $N \in\langle S(x)\rangle$. By condition (ii), $\Gamma_{N} \subset Q(x)$. Hence, $y \in \Gamma_{N} \subset Q(x)$, which is a contradiction. 
By Lemma 2.2, there exists $x_{0} \in \bigcap_{y \in Y} \overline{S^{*}(y)}$. By condition (i), there exists $y_{0} \in Y$ such that $x_{0} \in \operatorname{int} S^{-}\left(y_{0}\right)$. Hence, there exists a neighborhood $V$ of $x_{0}$ such that $V \subset S^{-}\left(y_{0}\right)$. It follows that $y_{0} \in S(x)$ for each $x \in V$. On the other hand, since $x_{0} \in \overline{S^{*}\left(y_{0}\right)}$, we know that $V \cap S^{*}\left(y_{0}\right) \neq \emptyset$. Hence, there exists $x \in V$ such that $x \in S^{*}\left(y_{0}\right)$, and it follows $y_{0} \notin S(x)$, which is a contradiction. This completes the proof.

Remark 3.1. (a) If $Y$ is a convex space with $\Gamma=$ co and $S=Q$, then Theorem 3.1 reduces to Theorem 2.5 in Lin, Ansari and Wu [14]; (b) We would like to point out that the proof of Theorem 3.1 is quite different from the proof of Theorem 2.5 in [14].

Example 3.1 Let $X=[1,+\infty)$ be endowed with Euclidean topology and $Y=[0,+\infty)$ with $\Gamma=$ co. Let $S: X \multimap Y, Q: X \multimap Y$ and $T: Y \multimap X$ be three maps defined, respectively, by

and

$$
\begin{gathered}
S(x)=\{0\}, \quad \forall x \in X, \\
Q(x)=[0, x), \quad \forall x \in X
\end{gathered}
$$

$$
T(y)=\{1\}, \quad \forall y \in Y .
$$

Then we can check that all the conditions of Theorem 3.1 are satisfied.

(i) Since

we have

$$
S^{-}(y)= \begin{cases}X, & \text { if } y=0 \\ \emptyset, & \text { if } y>0\end{cases}
$$

$$
X=S^{-}(0)=\bigcup_{y \in Y} \operatorname{int} S^{-}(y)
$$

and so condition (i) of Theorem 3.1 is satisfied.

(ii) For each $x \in X$ and $M \in\langle S(x)\rangle$, we have $M=\{0\}$ and so

$$
\Gamma_{M}=\operatorname{co} M=\{0\} \subset[0, x)=Q(x) .
$$

It follows that condition (ii) of Theorem 3.1 is satisfied.

(iii) For any KKM map $F: Y \multimap X$ with respect to $T$, we have $T\left(\Gamma_{A}\right) \subset$ $F(A)$ for each $A \in\langle Y\rangle$, i.e., $1 \in F(A)$ for each $A \in\langle Y\rangle$. Hence $1 \in \bigcap_{y \in Y} F(y)$, and it follows that $T \in \mathfrak{R}(Y, X) \subset \mathfrak{R C}(Y, X)$. Since $T(Y)=\{1\}$ and $\{1\}$ is compact, we know that $T$ is compact. This shows that condition (iii) of Theorem 3.1 is satisfied.

Thus, it follows from (i)-(iii) that all the conditions of Theorem 3.1 are satisfied.

When $T$ is not necessarily compact, we have the following result.

Theorem 3.2. Let $X$ be a topological space and $(Y ; \Gamma)$ be an abstract convex space. Let $S: X \multimap Y, Q: X \multimap Y$ and $T: Y \multimap X$ be three maps satisfying the following conditions:

(i) $X=\bigcup_{y \in Y}$ int $S^{-}(y)$; 
(ii) for each $x \in X, M \in\langle S(x)\rangle$ implies $\Gamma_{M} \subset Q(x)$;

(iii) $T \in \mathfrak{R} \mathfrak{C}(Y, X)$;

(iv) for each compact subset $A$ of $Y, \overline{T(A)}$ is compact;

(v) there exists a nonempty compact subset $D$ of $X$ such that for each $N \in\langle Y\rangle$, there exists a compact $\Gamma$-convex subset $L_{N}$ of $Y$ containing $N$ such that $T\left(L_{N}\right) \backslash D \subset \cup\left\{\operatorname{int} S^{-}(y): y \in L_{N}\right\}$.

Then there exists $\left(x_{0}, y_{0}\right) \in X \times Y$ such that $x_{0} \in T\left(y_{0}\right)$ and $y_{0} \in Q\left(x_{0}\right)$.

Proof. Since $D$ is compact and

$$
D \subset X=\bigcup_{y \in Y} \operatorname{int} S^{-}(y),
$$

there exists a finite subset $N \in\langle Y\rangle$ such that

$$
D \subset \cup\left\{\operatorname{int} S^{-}(y): y \in N\right\} .
$$

By condition (v), there exists a compact $\Gamma$-convex subset $L_{N}$ of $Y$ containing $N$ such that

$$
T\left(L_{N}\right) \backslash D \subset \cup\left\{\operatorname{int} S^{-}(y): y \in L_{N}\right\} .
$$

By (3.1), we get

$$
T\left(L_{N}\right) \cap D \subset \cup\left\{\operatorname{int} S^{-}(y): y \in N\right\} \subset \cup\left\{\operatorname{int} S^{-}(y): y \in L_{N}\right\} .
$$

From (3.2) and (3.3), we have

$$
T\left(L_{N}\right) \subset \cup\left\{\operatorname{int} S^{-}(y): y \in L_{N}\right\} .
$$

Since $(Y ; \Gamma)$ is an abstract convex space and $L_{N}$ is a $\Gamma$-convex subset of $Y$, $\left(L_{N} ;\left.\Gamma\right|_{\left\langle L_{N}\right\rangle}\right)$ is also an abstract convex space. By condition (iv), $\overline{T\left(L_{N}\right)}$ is compact, and hence $\left.T\right|_{L_{N}}$ is compact. Since $T \in \mathfrak{R C}(Y, X)$ and $L_{N}$ is a $\Gamma$-convex subset of $Y$, it follows from Lemma 2 in [18] that $\left.T\right|_{L_{N}} \in \mathfrak{R C}\left(L_{N}, T\left(L_{N}\right)\right)$. By Theorem 3.1, there exist $x_{0} \in T\left(L_{N}\right) \subset X$ and $y_{0} \in L_{N} \subset Y$ such that $\left.x_{0} \in T\right|_{L_{N}}\left(y_{0}\right)=T\left(y_{0}\right)$ and $\left.y_{0} \in Q\right|_{T\left(L_{N}\right)}\left(x_{0}\right)=Q\left(x_{0}\right)$. This completes the proof.

Remark 3.2. (a) If $Y$ is a convex space with $\Gamma=$ co and $S=Q$, then Theorem 3.2 reduces to Theorem 2.6 in Lin, Ansari and Wu [14]; (b) If $T$ is compact or $X$ is compact, then conditions (iv) and (v) become superfluous.

Example 3.2 Let $X=[1,+\infty)$ be endowed with Euclidean topology and $Y=[0,+\infty)$ with $\Gamma=$ co. Let $S: X \multimap Y, Q: X \multimap Y$ and $T: Y \multimap X$ be three maps defined, respectively, by

$$
\begin{gathered}
S(x)=\{0\}, \quad \forall x \in X, \\
Q(x)=[0, x), \quad \forall x \in X
\end{gathered}
$$

and

$$
T(y)=\{y+1\}, \quad \forall y \in Y .
$$

Then we can check that all the conditions of Theorem 3.2 are satisfied. 
(i) By Example 3.1(i), we have $X=\bigcup_{y \in Y}$ int $S^{-}(y)$ and so condition (i) of Theorem 3.2 is satisfied.

(ii) By Example 3.1(ii), for each $x \in X$ and $M \in\langle S(x)\rangle$, we have $\Gamma_{M} \subset$ $Q(x)$. So condition (ii) of Theorem 3.2 is satisfied.

(iii) It is known that for abstract convex space $(Y$; co) where $Y \subset \mathbb{R}$, the identity map $1_{Y} \in \mathfrak{R C}(Y, Y) \cap \mathfrak{R O}(Y, Y)$. Since $T: Y \multimap X$ is a continuous single-valued map, we have $T \in \mathfrak{R C}(Y, X) \cap \mathfrak{R O}(Y, X)$ by Lemma 4 in Park [18]. This shows that condition (iii) of Theorem 3.2 is satisfied.

(iv) Since $T$ is a continuous single-valued map, we have $T(A)$ is compact for each compact subset $A$ of $Y$. Hence, $\overline{T(A)}=T(A)$ is compact. This shows that condition (iv) of Theorem 3.2 is satisfied.

(v) Let $D=[1,2] \subset X=[1,+\infty)$. Then $D$ is nonempty compact. For each $N \in\langle Y\rangle$, let $L_{N}=[0, \max N]$. Obviously, $L_{N}$ is a compact convex subset of $Y$ containing $N$ such that $T\left(L_{N}\right) \backslash D \subset \cup\left\{\right.$ int $\left.S^{-}(y): y \in L_{N}\right\}=S^{-}(0)=X$. This shows that condition (v) of Theorem 3.2 is satisfied.

It follows from (i)-(v) that all the conditions of Theorem 3.2 are satisfied.

As a simple consequence of Theorem 3.2, we have the following fixed point result, which is indeed a proper generalization of Fan-Browder fixed point theorem [4].

Corollary 3.1. Let $(X ; \Gamma)$ be an abstract convex topological space with $1_{X} \in$ $\mathfrak{R C}(X, X)$. Let $S, Q: X \multimap X$ be two maps satisfying the following conditions:

(i) $X=\bigcup_{y \in X}$ int $S^{-}(y)$;

(ii) for each $x \in X, M \in\langle S(x)\rangle$ implies $\Gamma_{M} \subset Q(x)$;

(iii) there exists a nonempty compact subset $D$ of $X$ such that for each $N \in\langle X\rangle$, there exists a compact $\Gamma$-convex subset $L_{N}$ of $X$ containing $N$ such that $L_{N} \backslash D \subset \cup\left\{\operatorname{int} S^{-}(y): y \in L_{N}\right\}$.

Then there exists $x_{0} \in X$ such that $x_{0} \in Q\left(x_{0}\right)$.

Proof. The conclusion follows from Theorem 3.2 by letting $X=Y$ and $T(x)=$ $\{x\}$ for all $x \in X$. This completes the proof.

Remark 3.3. (a) For any $G$-convex space $(X ; \Gamma)$, since the identity map $1_{X} \in$ $\mathfrak{R C}(X, X) \cap \mathfrak{R O}(X, X)$, we know that if $(X ; \Gamma)$ is a $G$-convex space, then Corollary 3.1 reduces to Lemma 2 in Balaj and Lin [3]; (b) Corollary 3.1 also generalizes Theorem 4 in [26] from $G$-convex spaces to abstract convex topological spaces; (c) If $T$ is compact or $X$ is compact, then condition (iii) becomes superfluous.

Theorem 3.3. Let $X$ be a topological space, $(Y ; \Gamma)$ be an abstract convex space and $Z$ be a nonempty set. Let $F: X \multimap Z, P: Y \multimap Z, Q: X \multimap Y$ and $T: Y \multimap X$ be four maps satisfying the following conditions:

(i) $Q$ has $\Gamma$-convex values;

(ii) for each $x \in X,\{y \in Y: F(x) \cap P(y) \neq \emptyset\} \subset Q(x)$;

(iii) $X=\bigcup_{z \in P(Y)}$ int $F^{-}(z)$; 
(iv) $T \in \mathfrak{R C}(Y, X)$;

(v) for each compact subset $A$ of $Y, \overline{T(A)}$ is compact;

(vi) there exists a nonempty compact subset $D$ of $X$ such that for each $N \in\langle Y\rangle$, there exists a compact $\Gamma$-convex subset $L_{N}$ of $Y$ containing $N$ such that $T\left(L_{N}\right) \backslash D \subset \bigcup_{y \in L_{N}}$ int $\{x \in X: F(x) \cap P(y) \neq \emptyset\}$.

Then there exists $\left(x_{0}, y_{0}\right) \in X \times Y$ such that $x_{0} \in T\left(y_{0}\right)$ and $y_{0} \in Q\left(x_{0}\right)$.

Proof. Define the map $S: X \multimap Y$ by

$$
S(x)=\{y \in Y: F(x) \cap P(y) \neq \emptyset\}, \forall x \in X .
$$

Then

$$
S^{-}(y)=\{x \in X: F(x) \cap P(y) \neq \emptyset\}, \forall y \in Y .
$$

It is easy to see that for a family $\left\{A_{i}\right\}_{i \in I}$ of subsets of a topological space, $\bigcup_{i \in I} \operatorname{int} A_{i} \subset \operatorname{int}\left(\bigcup_{i \in I} A_{i}\right)$. Having this fact in mind we obtain

$$
\begin{aligned}
\bigcup_{y \in Y} \operatorname{int} S^{-}(y) & =\bigcup_{y \in Y} \operatorname{int}\{x \in X: F(x) \cap P(y) \neq \emptyset\} \\
& =\bigcup_{y \in Y} \operatorname{int}\left(\bigcup_{z \in P(y)} F^{-}(z)\right) \\
& \supset \bigcup_{y \in Y} \bigcup_{z \in P(y)} \operatorname{int} F^{-}(z) \\
& =\bigcup_{z \in P(Y)} \operatorname{int} F^{-}(z) .
\end{aligned}
$$

Thus, by condition (iii) it follows that $X=\bigcup_{y \in Y}$ int $S^{-}(y)$.

By condition (ii), for each $x \in X$ and $M \in\langle S(x)\rangle$ we have $M \in\langle Q(x)\rangle$. By condition (i), $\Gamma_{M} \subset Q(x)$. The conclusion follows from Theorem 3.2. This completes the proof.

Remark 3.4. If $T$ is compact or $X$ is compact, then conditions (v) and (vi) become superfluous.

Example 3.3 Let $X=[1,+\infty)$ be endowed with Euclidean topology, $Y=$ $[0,+\infty)$ with $\Gamma=$ co and $Z=[0,+\infty)$. Let $F: X \multimap Z, P: Y \multimap Z$, $Q: X \multimap Y$ and $T: Y \multimap X$ be four maps defined, respectively, by

$$
\begin{gathered}
F(x)=\{0\}, \quad \forall x \in X, \\
P(y)= \begin{cases}Z, & \text { if } y=0, \\
\emptyset, & \text { if } y>0,\end{cases} \\
Q(x)=[0, x), \quad \forall x \in X
\end{gathered}
$$

and

$$
T(y)=\{y+1\}, \quad \forall y \in Y .
$$

Then we can check that all the conditions of Theorem 3.3 are satisfied. 
(i) For each $x \in X, Q(x)=[0, x)$ is convex and so is $\Gamma$-convex. This shows that condition (i) of Theorem 3.3 is satisfied.

(ii) For each $x \in X,\{y \in Y: F(x) \cap P(y) \neq \emptyset\}=\{0\} \subset[0, x)=Q(x)$. This shows that condition (ii) of Theorem 3.3 is satisfied.

(iii) Since

$$
F^{-}(z)= \begin{cases}X, & \text { if } z=0 \\ \emptyset, & \text { if } z>0\end{cases}
$$

and $P(Y)=Z$, we have

$$
X=F^{-}(0)=\bigcup_{z \in Z} \operatorname{int} F^{-}(z)=\bigcup_{z \in P(Y)} \operatorname{int} F^{-}(z) .
$$

This shows that condition (iii) of Theorem 3.3 is satisfied.

(iv) By Example 3.2(iii), we have $T \in \mathfrak{R} \mathfrak{C}(Y, X)$. Thus condition (iv) of Theorem 3.3 is satisfied.

(v) By Example 3.2(iv), for each compact subset $A$ of $Y, \overline{T(A)}$ is compact. Thus condition (v) of Theorem 3.3 is satisfied.

(vi) Let $D=[1,2] \subset X=[1,+\infty)$. Then $D$ is nonempty compact. For each $N \in\langle Y\rangle$, let $L_{N}=[0, \max N]$. Obviously, $L_{N}$ is a compact convex subset of $Y$ containing $N$ such that

$$
\begin{aligned}
T\left(L_{N}\right) \backslash D & \subset \bigcup_{y \in L_{N}} \text { int }\{x \in X: F(x) \cap P(y) \neq \emptyset\} \\
& =\operatorname{int}\{x \in X: F(x) \cap P(0) \neq \emptyset\}=X .
\end{aligned}
$$

Thus condition (vi) of Theorem 3.3 is satisfied.

It follows from (i)-(vi) that all the conditions of Theorem 3.3 are satisfied.

Corollary 3.2. Let $(X ; \Gamma)$ be an abstract convex topological space with $1_{X} \in$ $\mathfrak{R C}(X, X)$ and $Z$ be a nonempty set. Let $F, P: X \multimap Z$ and $Q: X \multimap X$ be three maps satisfying the following conditions:

(i) $Q$ has $\Gamma$-convex values;

(ii) for each $x \in X,\{y \in X: F(x) \cap P(y) \neq \emptyset\} \subset Q(x)$;

(iii) $X=\bigcup_{z \in P(X)}$ int $F^{-}(z)$;

(iv) there exists a nonempty compact subset $D$ of $X$ such that for each $N \in\langle X\rangle$, there exists a compact $\Gamma$-convex subset $L_{N}$ of $X$ containing $N$ such that $L_{N} \backslash D \subset \bigcup_{y \in L_{N}}$ int $\{x \in X: F(x) \cap P(y) \neq \emptyset\}$.

Then there exists $x_{0} \in X$ such that $x_{0} \in Q\left(x_{0}\right)$.

Remark 3.5. (a) When $(X ; \Gamma)$ is a $G$-convex space, Corollary 3.2 reduces to Theorem 1 in Balaj and Lin [3]; (b) If $T$ is compact or $X$ is compact, then condition (iv) becomes superfluous.

In particular, when $Q(x)=\{y \in Y: F(x) \cap P(y) \neq \emptyset\}$, Theorem 3.3 reduces to the following result: 
Theorem 3.4. Let $X$ be a topological space, $(Y ; \Gamma)$ be an abstract convex space and $Z$ be a nonempty set. Let $F: X \multimap Z, P: Y \multimap Z$ and $T: Y \multimap X$ be three maps satisfying the following conditions:

(i) for each $x \in X,\{y \in Y: F(x) \cap P(y) \neq \emptyset\}$ is $\Gamma$-convex;

(ii) $X=\bigcup_{z \in P(Y)}$ int $F^{-}(z)$;

(iii) $T \in \mathfrak{R C}(Y, X)$;

(iv) for each compact subset $A$ of $Y, \overline{T(A)}$ is compact;

(v) there exists a nonempty compact subset $D$ of $X$ such that for each $N \in\langle Y\rangle$, there exists a compact $\Gamma$-convex subset $L_{N}$ of $Y$ containing $N$ such that $T\left(L_{N}\right) \backslash D \subset \bigcup_{y \in L_{N}}$ int $\{x \in X: F(x) \cap P(y) \neq \emptyset\}$.

Then there exists $\left(x_{0}, y_{0}\right) \in X \times Y$ such that $x_{0} \in T\left(y_{0}\right)$ and $F\left(x_{0}\right) \cap P\left(y_{0}\right) \neq \emptyset$.

Remark 3.6. If $T$ is compact or $X$ is compact, then conditions (iv) and (v) become superfluous.

Example 3.4 Let $X=[1,+\infty)$ be endowed with Euclidean topology, $Y=$ $[0,+\infty)$ with $\Gamma=$ co and $Z=[0,+\infty)$. Let $F: X \multimap Z, P: Y \multimap Z$ and $T: Y \multimap X$ be three maps defined, respectively, by

$$
\begin{aligned}
& F(x)=\{0\}, \forall x \in X, \\
& P(y)= \begin{cases}Z, & \text { if } y=0, \\
\emptyset, & \text { if } y>0\end{cases}
\end{aligned}
$$

and

$$
T(y)=\{y+1\}, \forall y \in Y .
$$

Then we can check that all the conditions of Theorem 3.4 are satisfied. In fact, for each $x \in X,\{y \in Y: F(x) \cap P(y) \neq \emptyset\}=\{0\}$ is convex, and so is $\Gamma$-convex. This shows that condition (i) of Theorem 3.4 is satisfied. Furthermore, by Example 3.3(iii), (iv), (v) and (vi), we know that conditions (ii), (iii), (iv) and (v) of Theorem 3.4 are satisfied.

Corollary 3.3. Let $(X ; \Gamma)$ be an abstract convex topological space with $1_{X} \in$ $\mathfrak{R C}(X, X)$ and $Z$ be a nonempty set. Let $F, P: X \multimap Z$ be two maps satisfying the following conditions:

(i) for each $x \in X,\{y \in X: F(x) \cap P(y) \neq \emptyset\}$ is $\Gamma$-convex;

(ii) $X=\bigcup_{z \in P(X)}$ int $F^{-}(z)$;

(iii) there exists a nonempty compact subset $D$ of $X$ such that for each $N \in\langle X\rangle$, there exists a compact $\Gamma$-convex subset $L_{N}$ of $X$ containing $N$ such that $L_{N} \backslash D \subset \bigcup_{y \in L_{N}}$ int $\{x \in X: F(x) \cap P(y) \neq \emptyset\}$.

Then there exists $x_{0} \in X$ such that $F\left(x_{0}\right) \cap P\left(x_{0}\right) \neq \emptyset$.

Remark 3.7. (a) When $(X ; \Gamma)$ is a $G$-convex space, Corollary 3.3 reduces to Theorem 2 in Balaj and Lin [3], which generalizes Theorem 8 of [2] and Theorem 3.6 of [10]; (b) If $T$ is compact or $X$ is compact, then condition (iii) becomes superfluous. 


\section{Alternative theorems and minimax inequalities}

Recall that a point $x_{0} \in X$ is a maximal element for a map $F: X \multimap Z$ if $F\left(x_{0}\right)=\emptyset$. From Theorem 3.4, we get the following alternative theorem concerning the existence of maximal elements.

Theorem 4.1. Let $X$ be a topological space, $(Y ; \Gamma)$ be an abstract convex space and $Z$ be a nonempty set. Let $F: X \multimap Z, P: Y \multimap Z$ and $T: Y \multimap X$ be three maps satisfying the following conditions:

(i) for each $x \in X,\{y \in Y: F(x) \cap P(y) \neq \emptyset\}$ is $\Gamma$-convex;

(ii) $F^{-}$is transfer open valued;

(iii) $T \in \mathfrak{R C}(Y, X)$;

(iv) for each compact subset $A$ of $Y, \overline{T(A)}$ is compact;

(v) there exists a nonempty compact subset $D$ of $X$ such that for each $N \in\langle Y\rangle$, there exists a compact $\Gamma$-convex subset $L_{N}$ of $Y$ containing $N$ such that $T\left(L_{N}\right) \backslash D \subset \bigcup_{y \in L_{N}}$ int $\{x \in X: F(x) \cap P(y) \neq \emptyset\}$;

(vi) for each $y \in Y$ and $x \in T(y)$, we have $F(x) \cap P(y)=\emptyset$.

Then at least one of the following assertions holds:

(a) There exists $x_{0} \in X$ such that $F\left(x_{0}\right)=\emptyset$;

(b) There exists $z_{0} \in Z$ such that $P^{-}\left(z_{0}\right)=\emptyset$.

Proof. Suppose the conclusion would be false. Then $F$ has nonempty values and $P(Y)=Z$. Thus, by Lemma 2.1 we have

$$
X=\bigcup_{z \in Z} \operatorname{int} F^{-}(z)=\bigcup_{z \in P(Y)} \operatorname{int} F^{-}(z) .
$$

By Theorem 3.4, there exists $\left(x_{0}, y_{0}\right) \in X \times Y$ such that $x_{0} \in T\left(y_{0}\right)$ and $F\left(x_{0}\right) \cap P\left(y_{0}\right) \neq \emptyset$. This contradicts condition (vi), and completes the proof.

Remark 4.1. If $T$ is compact or $X$ is compact, then conditions (iv) and (v) become superfluous.

Corollary 4.1. Let $(X ; \Gamma)$ be an abstract convex topological space with $1_{X} \in$ $\mathfrak{R C}(X, X)$ and $Z$ be a nonempty set. Let $F, P: X \multimap Z$ be two maps satisfying the following conditions:

(i) for each $x \in X,\{y \in X: F(x) \cap P(y) \neq \emptyset\}$ is $\Gamma$-convex;

(ii) $F^{-}$is transfer open valued;

(iii) there exists a nonempty compact subset $D$ of $X$ such that for each $N \in\langle X\rangle$, there exists a compact $\Gamma$-convex subset $L_{N}$ of $X$ containing $N$ such that $L_{N} \backslash D \subset \bigcup_{y \in L_{N}}$ int $\{x \in X: F(x) \cap P(y) \neq \emptyset\}$;

(iv) for each $x \in X, F(x) \cap P(x)=\emptyset$.

Then at least one of the following assertions holds:

(a) There exists $x_{0} \in X$ such that $F\left(x_{0}\right)=\emptyset$;

(b) There exists $z_{0} \in Z$ such that $P^{-}\left(z_{0}\right)=\emptyset$. 
Remark 4.2. (a) When $(X ; \Gamma)$ is a $G$-convex space, Corollary 4.1 reduces to Theorem 3 in Balaj and Lin [3]; (b) If $T$ is compact or $X$ is compact, then condition (iii) becomes superfluous.

Corollary 4.2. In Theorem 4.1, if we assume further that $P(Y)=Z$, then there exists $x_{0} \in X$ such that $F\left(x_{0}\right)=\emptyset$.

Corollary 4.3. Let $(X ; \Gamma)$ be an abstract convex topological space with $1_{X} \in$ $\mathfrak{R C}(X, X)$ and $F: X \multimap X$ be a map satisfying the following conditions:

(i) for each $x \in X, F(x)$ is $\Gamma$-convex;

(ii) $F^{-}$is transfer open valued;

(iii) there exists a nonempty compact subset $D$ of $X$ such that for each $N \in\langle X\rangle$, there exists a compact $\Gamma$-convex subset $L_{N}$ of $X$ containing $N$ such that $L_{N} \backslash D \subset \bigcup_{y \in L_{N}}$ int $F^{-}(y)$;

(iv) for each $x \in X, x \notin F(x)$.

Then there exists $x_{0} \in X$ such that $F\left(x_{0}\right)=\emptyset$.

Proof. The conclusion follows from Corollaries 4.1 and 4.2 by letting $X=Z$ and $P(x)=\{x\}$ for all $x \in X$.

Remark 4.3. If $T$ is compact or $X$ is compact, then condition (iv) becomes superfluous.

An abstract convex space $(E, D ; \Gamma)$ is said to be compact if $E$ is a compact topological space. From now on, for simplicity, we are mainly concerned with compact abstract convex space $(X ; \Gamma)$ having $1_{X} \in \mathfrak{R C}(X, X)$. For example, any compact $G$-convex space, any compact $H$-space, or any compact convex space is such a space.

From Corollary 3.2, we get the following alternative theorem.

Definition $4.1([6])$. Let $X$ be a topological space and $Z$ be a nonempty set. A function $f: X \times Z \rightarrow \overline{\mathbb{R}}=\mathbb{R} \cup\{ \pm \infty\}$ is said to be $\alpha$-transfer upper semicontinuous ( $\alpha$-transfer u.s.c.) in the first variable for some $\alpha \in \mathbb{R}$, if for each $(x, z) \in X \times Z$ with $f(x, z)<\alpha$ there exist a neighborhood $N(x)$ of $x$ and a point $z^{\prime} \in Z$ such that $f\left(x^{\prime}, z^{\prime}\right)<\alpha$ for all $x^{\prime} \in N(x)$. If $f$ is $\alpha$-transfer upper semicontinuous in the first variable for each $\alpha \in \mathbb{R}$, we say that $f$ is transfer upper semicontinuous in the first variable.

Theorem 4.2. Let $(X ; \Gamma)$ be a compact abstract convex space with $1_{X} \in$ $\mathfrak{R C}(X, X)$ and $Z$ be a nonempty set. Let $f, g: X \times Z \rightarrow \overline{\mathbb{R}}, h: X \times X \rightarrow \overline{\mathbb{R}}$ be three functions and $\alpha, \beta, \gamma$ be three real numbers such that

(i) for each $x \in X$, the set $\{y \in X: h(x, y)<\gamma\}$ is $\Gamma$-convex;

(ii) $f$ is $\alpha$-transfer u.s.c. in the first variable;

(iii) for any $x, y \in X$ and $z \in Z$, the following implication holds: $f(x, z)<\alpha$ and $g(y, z)<\beta \Rightarrow h(x, y)<\gamma$

(iv) $h(x, x) \geq \gamma$ for all $x \in X$.

Then at least one of the following assertions holds: 
(a) There exists $x_{0} \in X$ such that $f\left(x_{0}, z\right) \geq \alpha$ for all $z \in Z$.

(b) There exists $z_{0} \in Z$ such that $g\left(x, z_{0}\right) \geq \beta$ for all $x \in X$.

Proof. Let $F, P: X \multimap Z$ and $Q: X \multimap X$ be defined, respectively, by

$$
\begin{array}{ll}
F(x)=\{z \in Z: f(x, z)<\alpha\}, & \forall x \in X, \\
P(x)=\{z \in Z: g(x, z)<\beta\}, & \forall x \in X
\end{array}
$$

and

$$
Q(x)=\{y \in X: h(x, y)<\gamma\}, \quad \forall x \in X .
$$

Suppose that both assertions (a) and (b) were false, i.e.,

(1) for each $x \in X$ there is $z \in Z$ such that $f(x, z)<\alpha$;

(2) for each $z \in Z$ there is $x \in X$ such that $g(x, z)<\beta$.

By (2), $P(X)=Z$. Let $x \in X$. By (1) and (ii), there exists a neighborhood $N(x)$ of $x$ and $z^{\prime} \in Z$ such that $f\left(x^{\prime}, z^{\prime}\right)<\alpha$ for all $x^{\prime} \in N(x)$. Hence $x \in \operatorname{int} F^{-}\left(z^{\prime}\right)$. Since $x$ has been arbitrarily chosen, we infer that

$$
X=\bigcup_{z \in Z} \operatorname{int} F^{-}(z)=\bigcup_{z \in P(X)} \operatorname{int} F^{-}(z) .
$$

By (i), $Q(x)$ is $\Gamma$-convex for each $x \in X$. By (iii), for each $x \in X,\{y \in$ $X: F(x) \cap P(y) \neq \emptyset\} \subset Q(x)$. Taking into account Remark 3.5(b), Corollary 3.2 implies that there exists $x_{0} \in X$ such that $x_{0} \in Q\left(x_{0}\right)$. It follows that $h\left(x_{0}, x_{0}\right)<\gamma$, which contradicts with condition (iv). This completes the proof.

Remark 4.4. When $(X ; \Gamma)$ is a compact $G$-convex space, Theorem 4.2 reduces to Theorem 12 in Balaj and Lin [3].

Definition $4.2([18])$. For abstract convex space $(X ; \Gamma)$, a real function $f$ : $X \rightarrow \overline{\mathbb{R}}$ is said to be quasiconvex (resp. quasiconcave) if $\{x \in X: f(x)<r\}$ (resp. $\{x \in X: f(x)>r\}$ ) is $\Gamma$-convex for each $r \in \overline{\mathbb{R}}$.

From Theorem 4.2, we derive the following minimax inequality.

Theorem 4.3. Let $(X ; \Gamma)$ be a compact abstract convex space with $1_{X} \in$ $\mathfrak{R C}(X, X)$ and $Z$ be a nonempty set. Let $f, g: X \times Z \rightarrow \overline{\mathbb{R}}$ and $h: X \times X \rightarrow \overline{\mathbb{R}}$ be three functions such that

(i) $h(x, \cdot)$ is quasiconvex for each $x \in X$;

(ii) $f$ is transfer u.s.c. in the first variable;

(iii) for any $x, y \in X$ and $z \in Z, h(x, y) \leq f(x, z)+g(y, z)$.

Then

$$
\inf _{x \in X} h(x, x) \leq \sup _{x \in X} \inf _{z \in Z} f(x, z)+\sup _{z \in Z} \inf _{x \in X} g(x, z)
$$

with the convention $\infty+(-\infty)=\infty$. 
Proof. We may assume that

$$
\inf _{x \in X} h(x, x)>-\infty, \sup _{x \in X} \inf _{z \in Z} f(x, z)<+\infty, \sup _{z \in Z} \inf _{x \in X} g(x, z)<+\infty .
$$

Suppose the conclusion of Theorem 4.3 would be false, i.e.,

$$
\inf _{x \in X} h(x, x)>\sup _{x \in X} \inf _{z \in Z} f(x, z)+\sup _{z \in Z} \inf _{x \in X} g(x, z) .
$$

Choose $\alpha, \beta, \gamma \in \mathbb{R}$ such that

$$
\sup _{x \in X} \inf _{z \in Z} f(x, z)<\alpha, \sup _{z \in Z} \inf _{x \in X} g(x, z)<\beta, \inf _{x \in X} h(x, x)>\gamma \text { and } \alpha+\beta<\gamma .
$$

It is easy to verify that functions $f, g$ and $h$ satisfy all the conditions of Theorem 4.2. We prove that neither assertion (a) nor assertion (b) of the conclusion of Theorem 4.2 can be true. In fact, if (a) happens, then

$$
\alpha \leq \inf _{z \in Z} f\left(x_{0}, z\right) \leq \sup _{x \in X} \inf _{z \in Z} f(x, z),
$$

a contradiction. If (b) happens, then

$$
\sup _{z \in Z} \inf _{x \in X} g(x, z) \geq \inf _{x \in X} g\left(x, z_{0}\right) \geq \beta,
$$

a contradiction again. This completes the proof.

Remark 4.5. When $(X ; \Gamma)$ is a compact $G$-convex space, Theorem 4.3 reduces to Theorem 13 in Balaj and Lin [3].

\section{References}

[1] M. Balaj, Coincidence and maximal element theorems and their applications to generalized equilibrium problems and minimax inequalities, Nonlinear Anal. 68 (2008), no. 12, 3962-3971.

[2] _ Applications of two matching theorems in generalized convex spaces, Nonlinear Anal. Forum 7 (2002), no. 1, 123-130.

[3] M. Balaj and L. J. Lin, Fixed points, coincidence points and maximal elements with applications to generalized equilibrium problems and minimax theory, Nonlinear Anal. 70 (2009), no. 1, 393-403.

[4] F. E. Browder, The fixed point theory of multi-valued mappings in topological vector spaces, Math. Ann. 177 (1968), 283-301.

[5] T. H. Chang and C. L. Yen, KKM property and fixed point theorems, J. Math. Anal. Appl. 203 (1996), no. 1, 224-235.

[6] X. P. Ding, Coincidence theorems and equilibria of generalized games, Indian J. Pure Appl. Math. 27 (1996), no. 11, 1057-1071.

[7] K. Fan, Sur un théoréme minimax, C. R. Acad. Sci. Paris 259 (1964), 3925-3928.

[8] C. D. Horvath, Contractibility and generalized convexity, J. Math. Anal. Appl. 156 (1991), no. 2, 341-357.

[9] __ Extension and selection theorems in topological spaces with a generalized convexity structure, Ann. Fac. Sci. Toulouse Math. (6) 2 (1993), no. 2, 253-269.

[10] H. Kim and S. Park, Remarks on the KKM property for open-valued multimaps on generalized convex spaces, J. Korean Math. Soc. 42 (2005), no. 1, 101-110.

[11] B. Knaster, C. Kuratowski, and S. Mazurkiewicz, Ein Beweis des Fixpunktsatzes fur n-dimensionale Simplexe, Fund. Math. 14 (1929), 132-137. 
[12] M. Lassonde, On the use of KKM multifunctions in fixed point theory and related topics, J. Math. Anal. Appl. 97 (1983), 151-201.

[13] L. J. Lin, Applications of a fixed point theorem in G-convex spaces, Nonlinear Anal. 46 (2001),no. 5, Ser. A: Theory Methods, 601-608.

[14] L. J. Lin, Q. H. Ansari, and J. Y. Wu, Geometric properties and coincidence theorems with applications to generalized vector equilibrium problems, J. Optim. Theory Appl. 117 (2003), no. 1, 121-137.

[15] F. C. Liu, A note on the von Neumann-Sion minimax principle, Bull. Inst. Math. Acad. Sinica 6 (1978), no. 2, 517-524.

[16] S. Park, On generalizations of the KKM principle on abstract convex spaces, Nonlinear Anal. Forum 11 (2006), no. 1, 67-77.

[17] Comments on recent studies on abstract convex spaces, Nonlinear Anal. Forum 13 (2008), no. 1, 1-17

[18] Elements of the KKM theory on abstract convex spaces, J. Korean Math. Soc. 45 (2008), no. 1, 1-27.

[19] Equilibrium existence theorems in KKM spaces, Nonlinear Anal. 69 (2008), no. 12, 4352-4364.

[20] — Remarks on fixed points, maximal elements, and equilibria of economies in abstract convex spaces, Taiwanese J. Math. 12 (2008), no. 6, 1365-1383.

[21] _ Ninety years of the Brouwer fixed point theorem, Vietnam J. Math. 27 (1999), no. 3, 193-232.

[22] _ Elements of the KKM theory for generalized convex spaces, Korean J. Comput. Appl. Math. 7 (2000), no. 1, 1-28.

[23] _ Fixed point theorems in locally G-convex spaces, Nonlinear Anal. 48 (2002), no. 6, Ser. A: Theory Methods, 869-879.

[24] S. Park and H. Kim, Admissible classes of multifunctions on generalized convex spaces, Proc. Coll. Natur. Sci., Seoul Nat. Univ. 18 (1993), 1-21.

[25] G. Q. Tian, Generalizations of the FKKM theorem and the Ky Fan minimax inequality, with applications to maximal elements, price equilibrium, and complementarity, J. Math. Anal. Appl. 170 (1992), no. 2, 457-471.

[26] Z. T. Yu and L. J. Lin, Continuous selection and fixed point theorems, Nonlinear Anal. 52 (2003), no. 2, 445-455.

\section{MING-GE YANG}

DEPARTMENT OF MATHEMATiCS

LUOYANG NORMAL UNIVERSITY

LuOyang, Henan 471022, P. R. China

E-mail address: yang_mingge@sina.com

NAN-JING HUANG

Department of Mathematics

SiCHUAN UNIVERSITY

Chengdu, Sichuan 610064, P. R. China

E-mail address: nanjinghuang@hotmail.com 